\title{
Modelo para la creación del juego de realidad alternativa «El Plan de Gauss»: matemáticas, relatos y juegos en instituciones de educación superior
}

\begin{abstract}
Diego Darío López-Mera
Docente ocasional tiempo completo de la Institución Universitaria Antonio José Camacho (Cali, Colombia) dlopez@profesores.uniajc.edu.co | http://orcid.org/0000-0003-2902-5159
\end{abstract}

\begin{abstract}
Ana Catalina Archila-Gutiérrez
Docente hora cátedra de la Institución Universitaria Antonio José Camacho (Cali, Colombia) acarchila@profesores.uniajc.edu.co | http://orcid.org/0000-0002-4688-0280
\end{abstract}

Bryan Camilo Hernández-Montoya

Docente hora cátedra de la Institución Universitaria Antonio José Camacho (Cali, Colombia) bchernandez@profesores.uniajc.edu.co | http://orcid.org/0000-0002-4176-6382

Sandra Esther Suárez-Chávez

Docente ocasional tiempo completo de la Institución Universitaria Antonio José Camacho (Cali, Colombia) ssuarez@profesores.uniajc.edu.co | https://orcid.org/0000-0003-1632-6809

Eider Hernán Pérez-Rojas

Docente hora cátedra de la Institución Universitaria Antonio José Camacho (Cali, Colombia) eperez@profesores.uniajc.edu.co | https://orcid.org/0000-0001-7428-6900

\section{Extracto}

Los relatos y los juegos como herramientas didácticas se están utilizando cada vez más para apoyar procesos de enseñanza-aprendizaje. Desde hace algunos años, los juegos de realidad alternativa, una clase especial de juegos que integran relatos y tecnologías de la información y la comunicación (TIC), se están incorporando en ámbitos universitarios. En este artículo se explica el proceso que se utilizó para el diseño y desarrollo de un juego de realidad alternativa educativo para los cursos de matemáticas básicas en una institución universitaria.

Palabras clave: enseñanza universitaria; herramienta didáctica; juego de realidad alternativa educativo; juego educativo; matemáticas básicas; procesos de enseñanza-aprendizaje.

Fecha de entrada: 28-12-2020 / Fecha de revisión: 30-03-2021 / Fecha de aceptación: 19-04-2021

Cómo citar: López-Mera, D. D., Archila-Gutiérrez, A. C., Hernández-Montoya, B. C., Suárez-Chávez, S. E. y PérezRojas, E. H. (2021). Modelo para la creación del juego de realidad alternativa "El Plan de Gauss»: matemáticas, relatos y juegos en instituciones de educación superior. Tecnología, Ciencia y Educación, 20, 133-154. https://doi.org/10.51302/tce.2021.529 


\title{
Model for the creation of the alternate reality game «The Plan of Gauss»: mathematics, stories and games in higher education institutions
}

\author{
Diego Darío López-Mera \\ Ana Catalina Archila-Gutiérrez \\ Bryan Camilo Hernández-Montoya \\ Sandra Esther Suárez-Chávez \\ Eider Hernán Pérez-Rojas
}

\section{Abstract}

The stories and games as teaching tools are increasingly being applied to support teaching-learning processes. For some years, alternate reality games are a special kind of games that integrate stories and information and communication technologies (ICT), and they are being incorporated into university areas. This paper explains what procedure was employed to bring around an educational alternate reality game for a basic math course at a high education institute; this report documents the outcomes of a pilot testing done to the first semester students.

Keywords: basic mathematics; educational alternate reality game; instructional technology; math education; teaching-learning process; university teaching.

Citation: López-Mera, D. D., Archila-Gutiérrez, A. C., Hernández-Montoya, B. C., Suárez-Chávez, S. E. and Pérez-Rojas, E. H. (2021). Model for the creation of the alternate reality game «The Plan of Gauss»: mathematics, stories and games in higher education institutions. Tecnología, Ciencia y Educación, 20, 133-154. https://doi.org/10.51302/tce.2021.529 


\section{Sumario}

1. Introducción

2. Juegos de realidad alternativa: relatos, TIC y juegos en la educación

3. Elaboración del modelo

4. El juego de realidad alternativa «El Plan de Gauss»

5. Discusión

6. Conclusiones

Referencias bibliográficas 


\section{Introducción}

En concordancia con el siglo XXI y las formas de aprender de los sujetos adscritos a esta época, hay un desafío que la web y las tecnologías digitales le ponen a la educación para generar estrategias pedagógicas alternativas para los procesos de enseñanza y aprendizaje. En consecuencia, las instituciones educativas han comenzado a integrar las TIC en el aula en una interacción interdisciplinar para ayudar en lo posible a la enseñanza. Wikis, cursos en línea masivos y abiertos (massive online open courses [MOOC]), videotutoriales de YouTube, videojuegos serios y juegos de realidad alternativa son algunos escenarios que se incorporan cada vez más en los ámbitos educativos por su forma de hacer participar activamente al sujeto del aprendizaje.

Los juegos de realidad alternativa son juegos que integran relatos y TIC, y su propuesta al conocimiento científico y tecnológico radica en la ludificación de la enseñanza y el aprendizaje, es decir, en la utilización de elementos y dinámicas lúdicas que posibilitan la motivación, la resolución de problemas y la participación colaborativa con estudiantes (Martin et al., 2006; Whitton, 2008). En este orden de ideas es fundamental hacer énfasis en la importancia de la articulación de una tecnología adecuada en los procesos de aprendizaje de los estudiantes para potenciar la apropiación y construcción de

Las oportunidades que los juegos de realidad alternativa ofrecen a la educación van desde el fortalecimiento de habilidades investigativas, la adquisición de conocimientos técnicos y el análisis crítico de lo que se enseña en el aula hasta la experiencia pedagógica de encontrar diversas formas de solucionar problemas gracias a la participación en un juego conocimiento. Lo anterior hace referencia a las múltiples lecturas y formas participativas de aprender que pueden tener los estudiantes, fomentando y fortaleciendo, a través de la conversación, la lectura, la escritura, la imagen, el vídeo o la música, formas más inclusivas, creativas y propositivas de asociarse con un nuevo conocimiento. Ahora bien, las oportunidades que los juegos de realidad alternativa ofrecen a la educación van desde el fortalecimiento de habilidades investigativas, la adquisición de conocimientos técnicos y el análisis crítico de lo que se enseña en el aula hasta la experiencia pedagógica de encontrar diversas formas de solucionar problemas gracias a la participación en un juego (Gordon, 2015; Palmer y Petroski, 2016; Whitton, 2008).

De esta manera, el resultado que se espera es que los participantes adquieran conceptos y los relacionen entre sí. En la presente investigación, los conceptos que se van a adquirir y a relacionar son los concernientes a las matemáticas básicas. 
En el caso específico del aprendizaje de las matemáticas, este puede convertirse en un muro difícil de sobrepasar para muchos estudiantes colombianos. Los resultados de las pruebas PISA (programme for international student assessment) 2015 y 2018 en Colombia, en las que se evaluó el rendimiento de estudiantes de colegio de grado medio en materias como matemáticas, lenguaje y ciencia, no fueron alentadores y diagnosticaron la dificultad que tienen los jóvenes para hacer frente a los retos que exigen las sociedades modernas, en particular los asociados a la resolución de problemas inesperados, no rutinarios y de contextos poco familiares (Conde, 2017; Organización para la Cooperación y el Desarrollo Económicos [OCDE], 2016, 2019; Sanabria et al., 2020). Tal situación también se refleja en las pruebas estatales y no es un problema exclusivo de Colombia. En realidad, se trata de un problema generalizado para Latinoamérica (Rivas, 2015) y, precisamente, estudiantes con deficiencias en matemáticas son los que llegan a las instituciones de educación superior.

En vista de lo anterior, se propone una estrategia didáctica a partir de la puesta en ejecución de un juego de realidad alternativa, el cual contribuyó a la apropiación de conocimientos en el área de matemáticas en estudiantes de primer semestre de la Institución Universitaria Antonio José Camacho (UniCamacho) de Colombia. El potencial pedagógico del juego de realidad alternativa se basa en la posibilidad que tiene el docente de ser un mediador del aprendizaje, el cual propicia la autonomía del estudiante a través de la tecnología, a la vez que lo impulsa a lograr el desarrollo de su razonamiento cuantitativo a partir de narrativas interactivas y retos colaborativos.

El presente artículo describe el modelo del proceso realizado para el diseño y desarrollo del juego de realidad alternativa «El plan de Gauss» ${ }^{1}$ y la prueba piloto llevada a cabo con estudiantes universitarios de los cursos de matemáticas básicas de la UniCamacho.

\section{Juegos de realidad alternativa: relatos, TIC y juegos en la educación}

La industria del entretenimiento no solo crea y distribuye contenidos a los que se puede acceder a través de diversos medios, sino que también los mezcla. En los noventa, cuando apareció la web, los medios convergieron en un estado en el cual todo se entremezclaba y transformaba rápidamente, afectando, inclusive, a la forma de presentar, contar y diseñar historias (Jenkins, 2006). Desde luego, lo anterior exige espectadores, lectores o audiencias más activas y comprometidas con un ferviente deseo por investigar y, sobre todo, por colaborar; porque solo así podrán conocer más sobre el universo narrativo que tanto admiran (Palmer y Petroski, 2016).

1 La elección del título del juego tuvo dos motivos: primero, para rendir homenaje al famoso matemático y físico alemán, Johann Carl Friedrich Gauss, y segundo, porque uno de los personajes principales del juego también se apellida Gauss y tiene una predisposición por crear retos matemáticos. 
En un juego de realidad alternativa, las partes que componen el relato están dispersas a través de diversos medios y no se encuentran exclusivamente en el ciberespacio. En estos juegos lo realizado en el «entorno virtual» se mezcla con lo hecho en el «mundo real», en un esfuerzo por difuminar la línea que separa la realidad de la ficción. Así, por ejemplo, una actividad en el «mundo real» y en el ámbito educativo sería lo que podría suceder en un aula de clase; mientras que una actividad en el «entorno virtual» podrían ser las acciones llevadas a cabo por los estudiantes en redes sociales online o en videojuegos.

Ahora bien, los juegos, y, específicamente, la mayoría de los videojuegos, sumergen al jugador en una historia, en ocasiones durante horas y semanas. Tal es la cantidad de tiempo libre que muchas personas dedican voluntariamente a los juegos, que existen estudios sobre cómo incorporar y redireccionar estas actividades lúdicas en entornos que comúnmente no lo son (De Aguilera y Mendiz, 2003; Barr, 2017; García, 2009). Según Ramírez (2014), por ejemplo, cuando una empresa ludifica algunos de sus procesos puede lograr un mejor desempeño de sus trabajadores o captar y fidelizar clientes. Asimismo, cada vez más aparecen juegos y videojuegos que pretenden, además de entretener, ser herramientas didácticas, y esto está sucediendo con los juegos de realidad alternativa.

Una curiosidad implícita en los juegos de realidad alternativa, y que se convierte en su característica diferenciadora del resto de juegos, es su autonegación a considerarse un juego como tal. En sus distintas definiciones, aparece la frase «this is not a game» como recordatorio para que los diseñadores de los juegos de realidad alternativa (también conocidos como puppet masters) se esfuercen más en difuminar esa línea que separa la realidad del jugador con la ficción del juego (De Beer, 2016; McGonigal, 2003). Esto implica que los jugadores no asuman ni interpreten a un personaje (por el contrario, la pretensión es que sean ellos mismos durante el juego) y que la puerta de ingreso al juego, que se conoce como «agujero de conejo» (rabbit hole), forme parte de la cotidianidad del jugador.

Otro aspecto interesante que hay que tener en cuenta es que, aunque los juegos de realidad alternativa se pueden practicar de manera individualizada, la tendencia es que se jueguen en colaboración con otros participantes, con el fin de superar los retos (Fujimoto, 2010).

Hay que tener en cuenta que, aunque los juegos de realidad alternativa se pueden practicar de manera individualizada, la tendencia es que se jueguen en colaboración con otros participantes, con el fin de superar los retos

Todas estas características convierten a los juegos de realidad alternativa en herramientas para ser analizadas y orientadas a propósitos educativos. A continuación, se describen algunos juegos de realidad alternativa que son utilizados en el ámbito de la educación:

- AGOG. Se diseñó para el desarrollo del pensamiento crítico hacia la interpretación de la historia y fue financiado con fondos de la National Science Foundation. Es un juego que mezcla la ficción con un incendio que realmente sucedió en 1877 , 
el cual ocasionó la destrucción de miles de modelos de patentes. Así que el jugador tiene la misión de rescatar artefactos retrofuturistas que se salvaron del siniestro (Bonsignore et al., 2012).

- ARGuing. Fue diseñado para el aprendizaje de idiomas y la diversidad lingüística en la enseñanza secundaria en Europa a través de las tecnologías de la Web 2.0. En este juego, los participantes tienen la misión de construir, a través del esfuerzo colectivo, una particular torre de Babel, como un lugar cimentado en la democracia, la tolerancia, la libertad, el acceso a la educación y el deseo de comunicarse e interactuar con personas de otras culturas (Connolly et al., 2011).

- Evoke. Desarrollado por el Instituto del Banco Mundial con el propósito de promover la visión del cambio global a través de la innovación social y motivar a los jóvenes, especialmente a los africanos, para que crearan y lideraran soluciones a problemas sociales críticos (pobreza, educación, energía sostenible, agua potable, conflictos, cambio climático, falta de asistencia médica y derechos humanos) (Waddington, 2013).

- Juego de realidad alternativa de la University of Washington Information School. Esta institución auspició el diseño de un juego de realidad alternativa para que los estudiantes de informática incorporaran valores éticos en un dispositivo que permitiera la comunicación con una extraterrestre extraviada en la Tierra (Eschler y Fullerton, 2014).

- Reality Ends Here. Se diseñó para capacitar y motivar a los estudiantes de primer año de la Escuela de Artes Cinematográficas de la University of Southern California en la elaboración de historias (Watson, 2012).

Así que la inclusión de los juegos de realidad alternativa y los juegos, en general, en los procesos de enseñanza y aprendizaje ya se está llevando a cabo en diversas instituciones de educación superior.

La potencialidad del juego se encuentra en que es un activador de la atención y una herramienta complementaria para usar en las aulas. Algunos juegos pueden ser digitales y otros, analógicos. Sin embargo, estos juegos deben tener una intencionalidad educativa y haber sido desarrollados mediante un proceso sistémico que se inicie con la especificación del objetivo de aprendizaje (Lozada-Ávila y Betancur-Gómez, 2017; Marfisi-Schottman et al., 2010; Marne et al, 2012).

\section{Elaboración del modelo}

La investigación se inició con una revisión documental de 13 juegos de realidad alternativa, específicamente orientados a la educación: Bonsignore et al. (2012); Carolei y Schlemmer 
(2015); Connolly et al. (2011); Donald (2008); Dondlinger (2009); Eschler y Fullerton (2014); Evans (2014); Jagoda et al. (2015); Jerrett (2016); Johnston et al. (2012); Ryan (2014); Waddington (2013) y Watson (2012).

El criterio de selección de estos juegos fue que en la documentación recopilada se encontraran detalles del proceso de diseño y desarrollo. Una vez seleccionados estos documentos se procedió a elaborar, para cada juego, una ficha técnica con los siguientes datos: título, año y lugar de ejecución, duración, propósito y sinopsis del juego de realidad alternativa, asignatura y área de conocimiento donde se aplica, conceptos del curso tratados en el juego, descripción de la jugabilidad y del agujero de conejo, perfil de los jugadores y otros participantes, medios utilizados y metodología usada en su elaboración.

Una vez realizado lo anterior se procedió a identificar las características comunes de todos los juegos en cuanto a procesos de diseño y desarrollo.

Los investigadores de esos juegos hicieron entrevistas o conformaron grupos focales con los docentes del área de conocimiento o de los cursos para los cuales se diseñó el juego de realidad alternativa. Estas aportaciones se utilizaron para la elaboración de la historia y el diseño de los retos. También se encuestó a los estudiantes que participaron como jugadores en el juego de realidad alternativa y se tuvieron en cuenta los comentarios de los que ya habían aprobado el curso.

Los juegos de realidad alternativa se propusieron como actividades en las que el alumnado podía ganar puntos extra en sus asignaturas u otro tipo de premios, como asistir a conferencias o recibir asesorías de profesionales especializados en el área de conocimiento.

Estas características fueron el punto de partida de la construcción de un proceso que permitiera la elaboración de un juego de realidad alternativa educativo.

En el diseño del juego de realidad alternativa «El plan de Gauss» y de la estrategia didáctica que lo respalda, se empleó una bitácora de sistematización de los aspectos más relevantes del Proyecto Educativo Institucional (PEI)², del modelo pedagógico y del currículo de los cursos de matemáticas de primer semestre de los programas tecnológicos de la UniCamacho, con los cuales se diseñó una secuencia didáctica. En otras palabras, para el diseño del juego de realidad alternativa no solo se tuvieron en cuenta los conceptos y contenidos del curso, sino además aspectos propios de la cultura del centro educativo en el cual se iba a utilizar el juego.

2 De acuerdo con la Ley general de educación de Colombia, el PEI debe ser elaborado por cada establecimiento educativo y concertado con la comunidad académica con el fin de que se convierta en una carta de navegación en la que se «especifiquen, entre otros aspectos, los principios y fines del establecimiento, los recursos docentes y didácticos disponibles y necesarios, la estrategia pedagógica, el reglamento para docentes y estudiantes y el sistema de gestión» (art. 73). 
El modelo pedagógico de la UniCamacho tiene una concepción humanista en la que, retomando a De Zubiría (2006), se busca y exige comprender a la sociedad y al ser humano en su multidimensionalidad, complejidad e integridad. En este sentido, se reconoce al hombre como ser individual, social y ecológico y a la sociedad como un sistema en el que interactúan continuamente las dualidades pensar-global/actuar-local y pensar-local/actuar-global (UniCamacho, 2013). Es un modelo orientado al pensamiento sistémico y complejo que reconoce un principio de no completitud y de incertidumbre en el pensamiento (Morin, 2009). Además, el modelo considera unos componentes prácticos para el aprendizaje, como son el aprendizaje autónomo, significativo (Ausubel, 1968) y colaborativo (Áreas et al., 2005).

Precisamente, esta orientación hacia un pensamiento sistémico y complejo, unido al fomento del comportamiento colaborativo, coincide con las actuaciones que los jugadores deben tener para jugar un juego de realidad alternativa en el que se enfrentarán a la reconstrucción de un relato fragmentado. Así que el estudiante-jugador ya no es un simple espectador o receptor. Tiene que esforzarse en ordenar el aparente desorden y relacionar la parte y el todo.

Por consiguiente, en el proceso planteado para la construcción del juego de realidad alternativa se tuvo especial cuidado en la elaboración de una historia fragmentada con jugabilidad colaborativa, de tal modo que estuviese en concordancia con los contenidos y conceptos matemáticos del curso donde se iba a poner en práctica. La historia se contextualizó de acuerdo a la cotidianidad de los estudiantes participantes y a la realidad social del país. Para lograr lo anterior, se plantearon y se llevaron a cabo dos subprocesos que se ejecutaron en paralelo y que fueron interdependientes:

- Educativo.

- Narrativo-lúdico.

El logro de un equilibrio entre estos subprocesos fue crítico, ya que uno de los puntos en contra que tienen los juegos y videojuegos utilizados en educación es que los jugadores pueden percibirlos como juegos diferentes a los que acostumbran a jugar, entendiéndose por «diferentes» como «aburridos» (Young, 2010). Por otra parte, también se tuvo especial cuidado en no desviarse al extremo opuesto, es decir, que el propósito del juego fuera solo el entretenimiento y dejar de lado su propósito como herramienta didáctica. En otras palabras, se consideró que un juego de realidad alternativa aplicado a la educación debería lograr que los jugadores tuvieran una experiencia de juego divertida y didáctica a la vez.

\author{
Un juego de realidad alternativa \\ aplicado a la educación debería \\ lograr que los jugadores tuvieran \\ una experiencia de juego divertida \\ y didáctica a la vez
}


La existencia de estos dos subprocesos, en lugar de uno, también se produjo porque se identificó la participación de dos equipos de especialistas con sus propias dinámicas de trabajo. En el subproceso educativo son importantes las reflexiones y los aportes de pedagogos y expertos en la temática del curso; y en el subproceso narrativo-lúdico se hace necesario el trabajo de escritores, diseñadores, artistas y expertos en diseño de estrategias de juego. Pero todos, desde luego, trabajando de forma colaborativa, manteniéndose siempre en comunicación y llegando a acuerdos. La figura 1 muestra la representación del proceso propuesto.

Figura 1. Modelo del proceso utilizado en el diseño y desarrollo del juego de realidad alternativa «El Plan de Gauss"

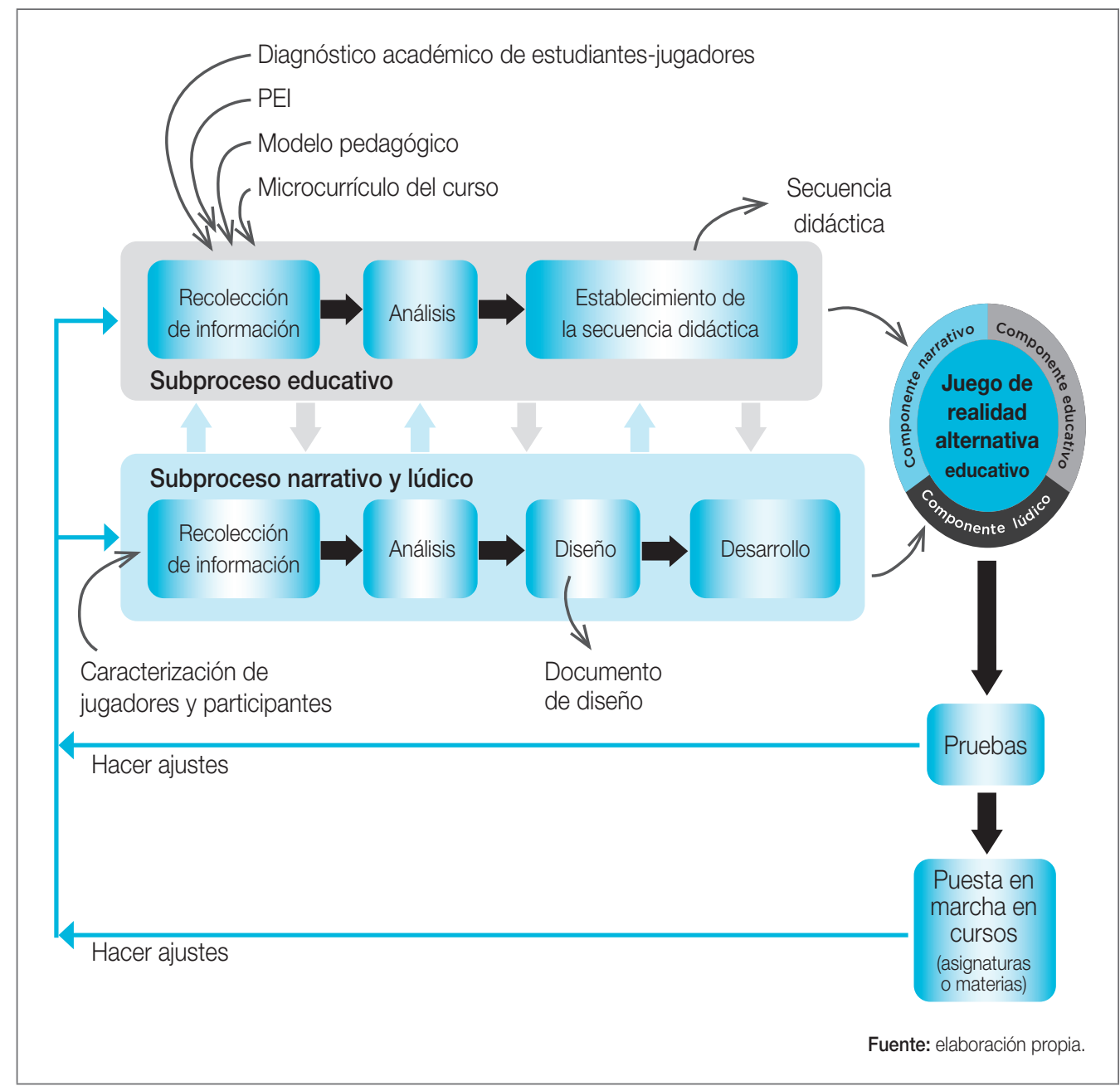


Centrándonos en lo planteado en la figura 1, el subproceso educativo contempla el diseño de una estrategia didáctica basada en un juego de realidad alternativa que tome en cuenta el PEl, el modelo pedagógico y el currículo del curso, así como la recolección y el análisis de información sobre la caracterización en cuanto al conocimiento matemático de la población objetivo, es decir, los estudiantes y los docentes. Este subproceso termina con el diseño de una secuencia didáctica que incorpora estos insumos, pero que simultáneamente considera e incluye los aportes del subproceso narrativo-lúdico.

En la fase de análisis del subproceso educativo se determina la estrategia didáctica que se relaciona más con el modelo pedagógico y con el PEl. De igual modo se establecen los objetivos de aprendizaje de la estrategia, en función de la identificación y de la selección de los conceptos matemáticos que requiere desarrollar el alumnado, de acuerdo al diseño curricular y a las dificultades y fortalezas que presentan los estudiantes con relación a las matemáticas.

En cuanto al subproceso narrativo-lúdico, su propósito es la creación del universo narrativo y de la historia del juego de realidad alternativa, así como el desarrollo de retos que tienen su base en las especificaciones obtenidas del subproceso educativo.

El subproceso narrativo-lúdico se inicia con un análisis de las características de los jugadores, es decir, de los estudiantes, tanto en lo individual como en lo colectivo. Esta se realiza para identificar los intereses de los potenciales usuarios, en cuanto a medios de comunicación y predilección por contenidos mediáticos. Una caracterización similar también se realiza con los profesores, es decir, con los otros participantes del juego de realidad alternativa.

En la fase de diseño del subproceso narrativo-lúdico se definió la historia en función de las características de los jugadores, así como de su estructura y universo narrativo. Todo se consignó en un documento de diseño en el que se registraron los resultados de los análisis, los acuerdos y la integración tanto del subproceso educativo como del narrativo-lúdico en cuanto a tecnología y medios que se iban a utilizar, los criterios de diseño de niveles y retos, los elementos artísticos y todo lo que se quería comunicar.

Por último, se realizó una prueba piloto con 16 estudiantes voluntarios de los primeros semestres. Los participantes fueron encuestados antes y después de la prueba con un cuestionario en escala de Likert. Las preguntas estaban orientadas a indagar sobre la utilidad e importancia del uso e incorporación de este tipo de juegos en cursos universitarios.

\section{El juego de realidad alternativa «El Plan de Gauss»}

Tomando como base el modelo, se diseñó el juego de realidad alternativa «El plan de Gauss» como herramienta para una estrategia didáctica centrada en los juegos. El cuadro 1 muestra la ficha técnica de este juego de realidad alternativa. 


\section{Cuadro 1. Ficha técnica del juego de realidad alternativa «El plan de Gauss»}

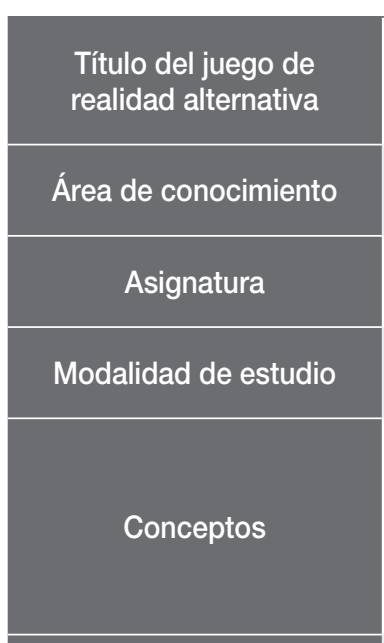

Propósito
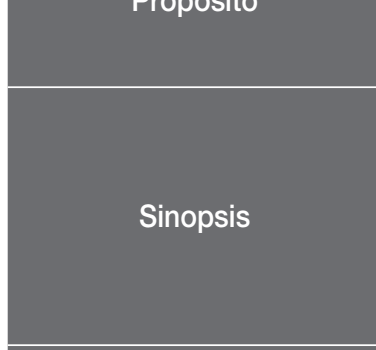

Agujero de conejo

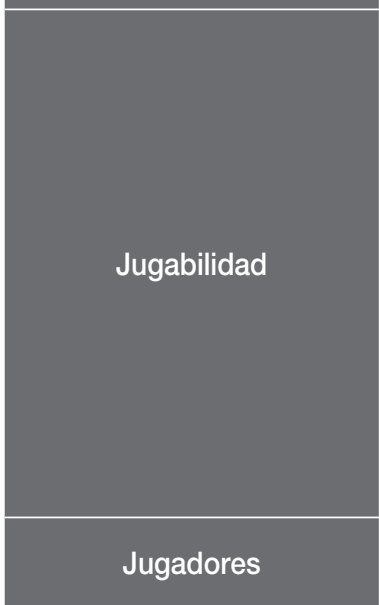

El Plan de Gauss.

Matemáticas.

Matemáticas 1.

Presencial.

Relación matemática.

Función matemática.

Función lineal.

Función cuadrática.

Es un juego de realidad alternativa para ser utilizado en la enseñanza y en el aprendizaje de las matemáticas básicas en instituciones de educación superior.

Angie, Sara y Juan son estudiantes universitarios de primer semestre que coinciden en la asignatura Matemáticas 1. Llegan a ser buenos amigos; sin embargo, Angie desaparece. Nadie sabe qué le sucedió ni dónde está, así que Sara y Juan se apresuran a buscarla, enfrentándose a sus propios temores y siendo acechados por un enemigo oculto.

Enlaces a vídeos noticiosos y al blog de Angie desde el blog del docente.

En cada una de las clases se plantea un reto para ser trabajado en grupo preferiblemente. Los retos, en su mayoría, consisten en problemas matemáticos contextualizados con lo que les sucede a los personajes de la historia. Algunos son para resolverse offline y otros, online, mediante el envío de las respuestas a través de formularios.

También hay un par de retos diseñados para que los estudiantes formen equipos y compitan. Uno trata sobre la identificación de coordenadas en un plano cartesiano, usando sensores de movimiento, y el otro consiste en un juego tipo concurso televisivo, en el cual se hacen preguntas de opción múltiple y el equipo que conteste primero va sumando puntos.

Todos los retos se evalúan con nota numérica.

Estudiantes de primer semestre de tecnologías. 
Participantes

Duración aproximada

Medios utilizados

Lugar de ejecución

Ciudad/País

Entidad desarrolladora

Observaciones
Estudiantes y docente de matemáticas.

8 semanas.

Blogs y sitio web de los personajes, blog del curso de matemáticas, página de Facebook de uno de los personajes, Gaceta Financiera (documentos PDF), videotutorial con la temática de relación matemática, vídeo noticioso, cortometrajes animados, cómics, novelas visuales, etc.

UniCamacho

Cali/Colombia.

UniCamacho

Este juego de realidad alternativa está planeado para ser utilizado en una parte de las clases de la asignatura Matemáticas 1. Se estima que la duración de las actividades varía entre 1 y 1,5 h.

La historia es contada, principalmente, a partir de las publicaciones de los personajes en sus respectivos blogs. Es decir, ellos narran y publican su día a día, tanto lo que les sucede en la universidad como lo que les pasa en sus hogares y en otros escenarios.

Fuente: elaboración propia.

El juego de realidad alternativa «El plan de Gauss» se estructuró a partir del número de clases de la asignatura Matemáticas 1, es decir, en función de la cantidad de encuentros presenciales entre el docente y los estudiantes.

De este modo, antes de cada clase, que en este caso fueron ocho en total, se hicieron publicaciones en los blogs de los personajes con el fin de ir dando avances de la historia del juego de realidad alternativa de forma dosificada. En el cuadro 2 se muestra una parte de esta estructura, que se corresponde con las actividades realizadas en la segunda clase.

Una vez diseñado y desarrollado el juego de realidad alternativa, se hizo una prueba piloto con estudiantes que participaron de forma voluntaria. En el cuadro 3 se muestra una comparativa entre la expectativa de los estudiantes al iniciar el curso y lo que experimentaron al terminarlo utilizando el juego de realidad alternativa.
El juego de realidad alternativa «El plan de Gauss» se estructuró a partir del número de clases de la asignatura Matemáticas 1, es decir, en función de la cantidad de encuentros presenciales entre el docente y los estudiantes 


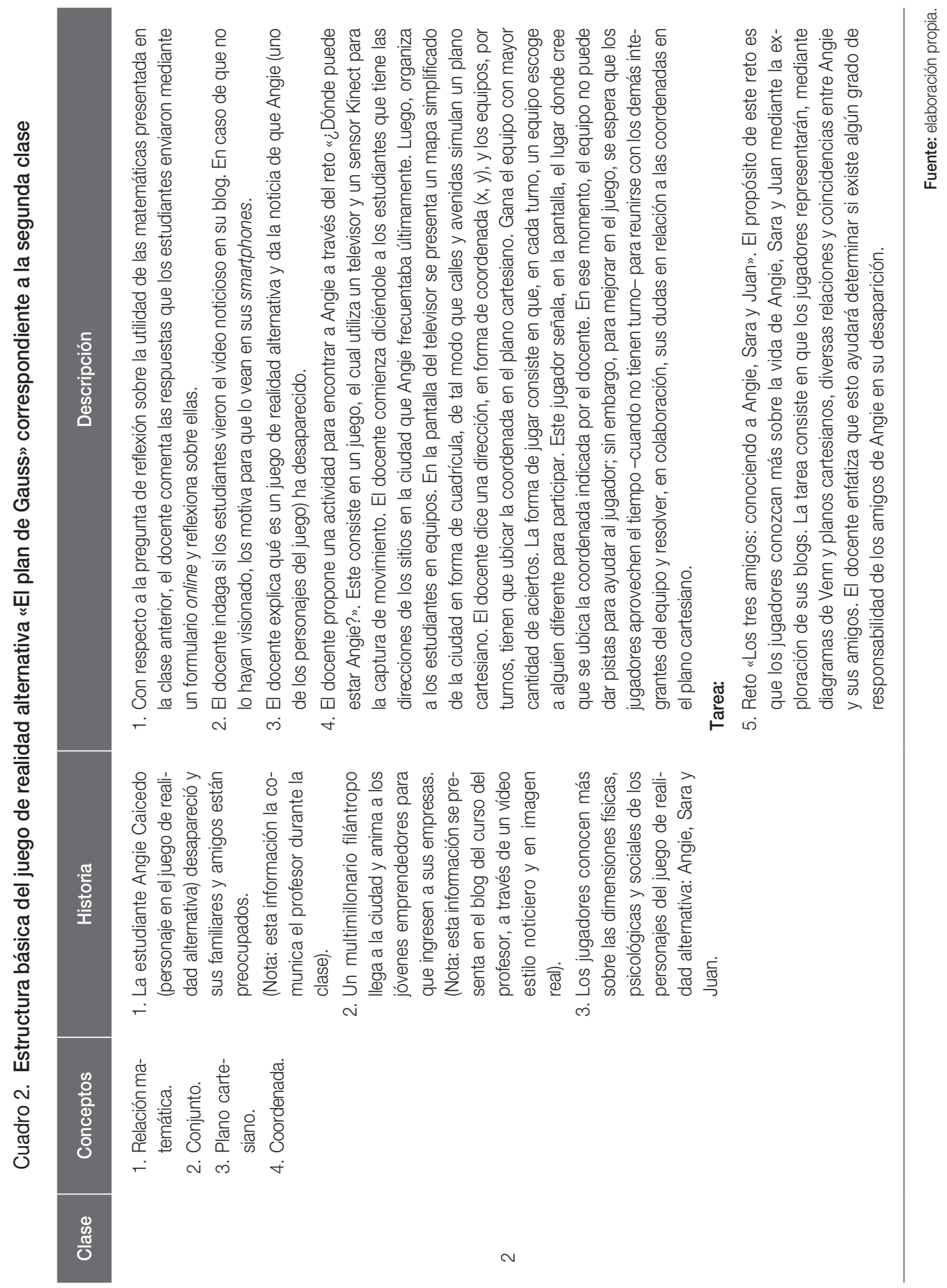


Cuadro 3. Expectativas de los estudiantes al inicio del curso versus curso finalizado

\begin{tabular}{|c|c|c|c|c|}
\hline Respuesta a la pregunta 1 & $\begin{array}{l}\text { Totalmente en } \\
\text { desacuerdo }\end{array}$ & En desacuerdo & De acuerdo & $\begin{array}{l}\text { Totalmente de } \\
\text { acuerdo }\end{array}$ \\
\hline Al inicio de la prueba piloto & $0 \%$ & $0 \%$ & $46 \%$ & $54 \%$ \\
\hline Al final de la prueba piloto & $0 \%$ & $0 \%$ & $27 \%$ & $73 \%$ \\
\hline \multicolumn{5}{|c|}{ Pregunta 2. ¿Considero importante aprender matemáticas a través del juego? } \\
\hline Respuesta a la pregunta 2 & $\begin{array}{l}\text { Totalmente en } \\
\text { desacuerdo }\end{array}$ & En desacuerdo & De acuerdo & $\begin{array}{l}\text { Totalmente de } \\
\text { acuerdo }\end{array}$ \\
\hline Al inicio de la prueba piloto & $0 \%$ & $0 \%$ & $62 \%$ & $38 \%$ \\
\hline Al final de la prueba piloto & $0 \%$ & $0 \%$ & $23 \%$ & $77 \%$ \\
\hline
\end{tabular}

Pregunta 3. ¿Me siento o me sentí incómodo participando en juegos para aprender matemáticas?

\begin{tabular}{cccccc}
\hline Respuesta a la pregunta 3 & $\begin{array}{c}\text { Totalmente en } \\
\text { desacuerdo }\end{array}$ & En desacuerdo & De acuerdo & $\begin{array}{c}\text { Totalmente de } \\
\text { acuerdo }\end{array}$ \\
\hline Al inicio de la prueba piloto & $44 \%$ & $44 \%$ & $12 \%$ & $0 \%$ \\
Al final de la prueba piloto & $61 \%$ & $23 \%$ & $0 \%$ & $16 \%$ \\
\hline
\end{tabular}

Pregunta 4. ¿Dedicaría tiempo fuera de clase a realizar actividades relacionadas con el juego para el aprendizaje de las matemáticas?

\begin{tabular}{lccccc}
\hline Respuesta a la pregunta 4 & $\begin{array}{c}\text { Totalmente en } \\
\text { desacuerdo }\end{array}$ & En desacuerdo & De acuerdo & $\begin{array}{c}\text { Totalmente de } \\
\text { acuerdo }\end{array}$ \\
\hline Al inicio de la prueba piloto & $6 \%$ & $0 \%$ & $94 \%$ & $0 \%$ \\
Al final de la prueba piloto & $0 \%$ & $23 \%$ & $54 \%$ & $23 \%$ \\
\hline Pregunta 5. ¿Disfruto cuando participo en juegos para la solución de problemas de matemáticas? \\
\hline Respuesta a la pregunta 5 & $\begin{array}{c}\text { Totalmente en } \\
\text { desacuerdo }\end{array}$ & En desacuerdo & De acuerdo & $\begin{array}{c}\text { Totalmente de } \\
\text { acuerdo }\end{array}$ \\
\hline Al inicio de la prueba piloto & $0 \%$ & $0 \%$ & $37 \%$ & $63 \%$ \\
Al final de la prueba piloto & $0 \%$ & $15 \%$ & $15 \%$ & $70 \%$ \\
\hline
\end{tabular}




\section{Discusión}

La articulación de los juegos de realidad alternativa como elemento pedagógico y formativo es relativamente reciente $\mathrm{y}$, aunque cada juego es distinto y está configurado de acuerdo a los requerimientos específicos del contexto, todos conservan las características constitutivas de un juego de realidad alternativa y siguen procesos similares a la hora de ser diseñados. En realidad, la única diferencia sustancial entre el modelo de la figura 1 y los procesos seguidos en el diseño de los otros juegos investigados es que, en estos últimos, no se evidencia un estudio que tenga en cuenta a los jugadores en la creación de la historia del juego.

En el juego de realidad alternativa «El Plan de Gauss», la elección del género y la escritura del argumento estuvo en consonancia con los resultados del análisis de las características de los jugadores; mientras que, en los otros juegos, esto lo determinó, exclusivamente, el equipo de investigadores.

El modelo tiene dos subprocesos interdependientes: el educativo y el narrativo-lúdico. Esta separación, en retrospectiva, fue importante, ya que durante el diseño del juego de realidad alternativa «El plan de Gauss» hubo «atascos creativos» en ambos subprocesos que, la mayoría de las veces, no ocurrían al mismo tiempo, lo que permitió que, mientras se solucionaba el «atasco» en uno de ellos, se avanzara en el otro subproceso. Por otra parte, su no fusión es una forma de acentuar la importancia de que ambos subprocesos requieren de expertos con diferentes habilidades.

Desde luego, estos atascos también pueden ser de otra clase. Carolei y Schlemmer (2015) advierten acerca de la dificultad de lograr un acuerdo entre los investigadores involucrados en el proyecto, sobre todo en una dicotomía que parece poco reconciliable: educativo versus entretenido.

Otra de las dificultades en el diseño de un juego de realidad alternativa educativo es el logro del aprendizaje a través de la colaboración. Al respecto, Bonsignore et al. (2012) proponen el diseño de actividades considerando los siguientes tipos de colaboración:

- Responsabilidad individual. La colaboración del jugador para el éxito de su equipo.

- Interdependencia positiva. La colaboración de los equipos para el éxito de todos.

- Interacción promotora. Los jugadores fomentan el esfuerzo de los demás, comparten recursos y hacen retroalimentación positiva.

- Procesamiento grupal. Los jugadores reflexionan sobre el juego y solucionan controversias. 
La falta de colaboración no solo puede detener el juego, sino ser fuente de conflictos. En el juego de realidad alternativa «El plan de Gauss» no se produjeron disputas; sin embargo, al inicio, sí hubo paradas de juego y, precisamente, esto sucedió por la falta de colaboración entre los participantes. Estos juegos visibilizan este tipo de dificultades.

\section{Los juegos de realidad alternativa, como herramientas, pueden utilizarse no solo para adquirir conocimientos sobre un tema, sino además para alcanzar un mayor desarrollo personal}

En consecuencia, como menciona Ryan (2014), los juegos de realidad alternativa, como herramientas, pueden utilizarse no solo para adquirir conocimientos sobre un tema, sino además para alcanzar un mayor desarrollo personal.

Ahora bien, haciendo un análisis de los aspectos técnicos del juego, específicamente a la forma de ingreso, los agujeros de conejo son insertados en la cotidianidad académica del alumnado como «pistas» que llevan a conocer la existencia de una historia. No obstante, estas pistas pueden pasar desapercibidas, a menos que se cuente con la intervención del docente como guía.

A pesar de que los estudiantes compiten en el juego, también colaboran con sus pares. Están inmersos en una jugabilidad en la que se les presentan retos que deben solucionar, usando sus competencias, habilidades y conocimientos aplicados. Todo a través de diversos medios que utilizan los puppet masters para dar a conocer la narrativa, comunicar los retos, generar encuentros entre los participantes y llevar un sistema de puntuación.

De esta forma, se hace evidente la integración de tres elementos para el adecuado funcionamiento de un juego de realidad alternativa en el ámbito educativo, los cuales, a su vez, están inmersos en los subprocesos educativo y narrativo-lúdico:

- La gamificación o ludificación en los procesos de enseñanza-aprendizaje como mecanismo para que el estudiante aprenda conceptos, fortalezca habilidades y, a través del reto o desafío, sienta la motivación para conseguir y alcanzar objetivos.

- El relato fragmentado y disperso en los medios adecuados con el propósito de generar el contexto de los retos del juego y presentar los conceptos clave del área de conocimiento.

- La generación de actividades orientadas al fortalecimiento de habilidades para el trabajo en equipo, aplicando los conceptos aprendidos a problemas representados en la cotidianidad y articulando lo aprendido en el aula en un contexto donde el esquema vertical de docente-estudiante se difumina. 
De todas formas, incorporar todo lo anterior al juego de realidad alternativa «El plan de Gauss» no fue fácil y habría sido aún más complicado si la comunicación entre los subprocesos no hubiese sido permanente y asertiva. Así que la fluida interdependencia y la constante comunicación entre los subprocesos educativo y narrativo-lógico se erigen como una condición fundamental para el éxito en el diseño y desarrollo de un juego de realidad alternativa educativo.
La fluida interdependencia y la constante comunicación entre los subprocesos educativo y narrativo-lógico se erigen como una condición fundamental para el éxito en el diseño y desarrollo de un juego de realidad alternativa educativo

Al final, en la prueba piloto, se constató que los estudiantes reclaman la inclusión de actividades lúdicas en los cursos, sobre todo si están contextualizadas y tienen una utilidad aplicable a los problemas cotidianos; no obstante, se descubrió que el alumnado percibía que el proceso era exigente, a pesar de que la herramienta didáctica utilizada fuese un juego. De este modo, en relación a las preguntas «¿Resolver retos matemáticos sobre problemas relacionados con la vida real resulta provechoso para mi aprendizaje?» « ¿Considero importante aprender matemáticas a través del juego?, los estudiantes están de acuerdo con la utilización de los juegos en los procesos de enseñanza-aprendizaje y, más aún, una vez que vivieron la experiencia con retos contextualizados en su día a día. A su vez, la respuesta generalizada a las preguntas « ¿Me siento o me sentí incómodo participando en juegos para aprender matemáticas?», «¿Dedicaría

En la prueba piloto, se constató que los estudiantes reclaman la inclusión de actividades lúdicas en los cursos, sobre todo si están contextualizadas y tienen una utilidad aplicable a los problemas cotidianos; no obstante, se descubrió que el alumnado percibía que el proceso era exigente, a pesar de que la herramienta didáctica utilizada fuese un juego tiempo fuera de clase para realizar actividades relacionadas con el juego para el aprendizaje de matemáticas?» $y$ « ¿Disfruto cuando participo en juegos para la solución de problemas de matemáticas?» fue positiva; no obstante, una vez concluido el curso, algunos estudiantes mostraron reparos en cuanto a su experiencia en el juego. En las observaciones realizadas en las clases se pudo identificar que algunos retos resultaban difíciles de superar $\sin$ la ayuda permanente del docente. Esto también sucedió con varios retos que se dejaron como tarea.

\section{Conclusiones}

La incorporación de los juegos en las clases potencia los procesos de enseñanzaaprendizaje de los estudiantes, por lo que su uso no debería destinarse únicamente a los más pequeños. Incluso se podría decir que las investigaciones de diseño y desarrollo de 
herramientas didácticas basadas en juegos deberían enfocarse más a los jugadores adultos. Es importante tener en cuenta que los juegos educativos no solo potencializan el saber y el saber hacer, sino además el saber ser.

El alumno, como futuro profesional, requiere de conocimientos y habilidades técnicas para desempeñar su trabajo, pero también de habilidades intra- e interpersonales; $y$, precisamente, con los juegos de realidad alternativa, los docentes cuentan con una herramienta extra para que sus estudiantes logren un desarrollo más integral.

\author{
La incorporación de los \\ juegos en las clases potencia los \\ procesos de enseñanza-aprendizaje \\ de los estudiantes, por lo que su \\ uso no debería destinarse \\ únicamente a los más pequeños. \\ Incluso se podría decir que \\ las investigaciones de diseño y \\ desarrollo de herramientas didácticas \\ basadas en juegos deberían enfocarse \\ más a los jugadores adultos
}

De todas formas, es conveniente que en el ámbito universitario los juegos sean percibidos como útiles e importantes tanto para el alumnado como para el profesorado. Además, tienen que estar diseñados de tal modo que representen un reto acorde con las habilidades de los estudiantes, para que estén más dispuestos a jugar; es decir, lo que se busca es que los jugadores permanezcan en un estado óptimo de experiencia interna o flow (flujo), como lo llamó Csikszentmihalyi (1990).

Aparte de lo anterior, puesto que la estrategia didáctica de la investigación está basada en un juego de realidad alternativa, se tienen que incluir, además, los objetivos de aprendizaje. En las entrevistas, los docentes de matemáticas, a pesar de que apoyaban la elaboración de nuevas herramientas didácticas, manifestaron su preocupación por la posible pérdida del rigor matemático. De este modo, bajo esta premisa, el juego de realidad alternativa debe contener y equilibrar adecuadamente los componentes narrativos, lúdicos y educativos, sin perder, por otra parte, la esencia de lo que es un juego de estas características. Un juego de realidad alternativa es, ante todo, un juego. Por consiguiente, dividir el proceso de creación de un juego de realidad alternativa educativo en dos subprocesos interdependientes fue acertado. En realidad, se trata de dos fuerzas que, en el proceso de diseño y desarrollo, tienen que hacerse oír y sentir, así como conseguir un adecuado balance.

Otro elemento esencial en un juego de realidad alternativa es su predisposición a ser colaborativo, es decir, que en dicho juego prevalezca la colaboración de los participantes frente a la competición. En esa difuminación de la realidad con la ficción del juego, los
Otro elemento esencial en un juego de realidad alternativa es su predisposición a ser colaborativo, es decir, que en dicho juego prevalezca la colaboración de los participantes frente a la competición. Los jugadores, para avanzar, resolver problemas y lograr metas satisfactorias tienen que colaborar con los demás


jugadores, tal y como sucede en la vida real, no son entidades solitarias, sino que viven en una sociedad en la que para avanzar, resolver problemas y lograr metas satisfactorias tienen que colaborar con los demás.

Así que lo interesante de los juegos de realidad alternativa que han sido diseñados para entornos educativos es que resultan ser estrategias y herramientas de apoyo para la enseñanza-aprendizaje, puesto que, para que los estudiantes tengan experiencias significativas con el objeto de estudio de los cursos, estos juegos se convierten en un medio de adquirir habilidades colaborativas y autoformativas, encontrando nuevas formas de relación e interacción social (entre sus pares y con el docente) y potencializando su formación a través del manejo de las TIC.

\section{Referencias bibliográficas}

Aguilera, M. de y Méndiz, A. (2003). Video games and education: (education in the face of a «parallel school»). CIE. Computers in Entertainment, 1(1), 1-10. https://doi. org/10.1145/950566.950583

Áreas Silva, J. D., Cárdenas Roa, C. y Estupiñán Farapuez, F. (2005). Aprendizaje cooperativo. Universidad Pedagógica Nacional.

Ausubel, D. (1968). Educational Psychology: A Cognitive View. Holt, Rinehart and Winston.

Barr, M. (2017). Video games can develop graduate skills in higher education students: a randomised trial. Computers \& Education, 113, 86-97. https://doi.org/10.1016/j.com pedu.2017.05.016

Beer, J. de. (2016). Analysing Alternate Reality Games Based on Game Design Theory to Propose a Conceptual Framework (Tesis doctoral, University of Pretoria). http://hdl. handle.net/2263/57176

Bonsignore, E., Hansen, D., Kraus, K., Ahn, J., Visconti, A., Fraistat, A. y Druin, A. (2012).
Alternate reality games: platforms for collaborative learning. En J. van Aalst, K. Thompson, M. J. Jacobson y P. Reimann (Eds.), The Future of Learning: Proceedings of the 10th International Conference of the Learning Sciences (pp. 251-258). https://reposi tory.isls.org//handle/1/2212

Carolei, P. y Schlemmer, E. (2015). Alternate reality game in museum: a process to construct experiences and narratives in hybrid context. Edulearn15 Proceedings (pp. 8.037-8.045).

Conde, R. (2017, octubre 10). La resolución de problemas en las matemáticas. Compartir Palabra Maestra. https://compartirpalabramaestra.org/articulos-informativos/la-resolucion-de-problemas-en-las-matematicas

Connolly, T., Stansfield, M. y Hainey, T. (2011). Alternate reality game for language learning: ARGuing for multilingual motivation. Computers \& Education, 57(1), 1389-1415. https:// doi.org/10.1016/j.compedu.2011.01.009 
Csikszentmihalyi, M. (1990). Flow: The Psychology of Optimal Performance. Harper \& Row.

Donald, J. (2008). The «blood on the stacks» ARG: immersive marketing meets library new student orientation. En A. Harris y S. Rice (Eds.), Gaming in Academic Libraries: Collections, Marketing, and Information Literacy (pp. 189-211). American Library Association. https://digitalcommons.trinity.edu/lib_ faculty/46

Dondlinger, M. (2009). The Global Village Playground: A Qualitative Case Study of Designing an $A R G$ as a Capstone Learning Experience (Tesis doctoral, University of North Texas). https://digital.library.unt.edu/ ark:/67531/metadc10995

Eschler, J. y Fullerton, S. (2014). Design planning for an alternate reality game to support learning of informatics concepts. iConference 2014 Proceedings (pp. 1.084-1.088). https://doi.org/10.9776/14388

Evans, E. (2014). «We're all a bunch of nutters!»: the production dynamics of alternate reality games. International Journal of Communication, 8(2014), 2.323-2.343. https://ijoc.org/ index.php/ijoc/article/view/2605

Fujimoto, R. (2010). Designing an Educational Alternate Reality Game. Shoyu Learning Solutions. http://www.shoyu.com/education/ Research_DesigningAnEducationalARG.pdf

García Gigante, B. (2009). Videojuegos: medio de ocio, cultura popular y recurso didáctico para la enseñanza y aprendizaje de las matemáticas escolares (Tesis doctoral, Universidad Autónoma de Madrid). http://hdl. handle.net/10486/3722

Gordon, R. (2015). Alternate Reality Games for Behavioral and Social Science Research. Carnegie Mellon University. https://doi.org/ 10.1184/R1/6686714.v1
Jagoda, P., Gilliam, M., McDonald, P. y Russell, C. (2015). Worlding through play: alternate reality games, large-scale learning, and the source. American Journal of Play, 8(1), 74-100.

Jenkins, H. (2006). Convergence Culture: Where Old and New Media Collide. New York University Press.

Jerrett, M. (2016). Using an Alternate Reality Game to Teach Information Literacy (MIS Dissertation, University of Pretoria). http:// hdl.handle.net/2263/57484

Johnston, J., Massey, A. y Marker, R. (2012). Using an alternate reality game to increase physical activity and decrease obesity risk of college students. Journal of Diabetes Science and Technology, 6(4), 828-838. https://doi. org/10.1177/193229681200600414

Lozada-Ávila, C. y Betancur-Gómez, S. (2017). La gamificación en la educación superior: una revisión sistemática. Revista Ingenierías Universidad de Medellin, 16(31), 97-124.

Marfisi-Schottman, I., George, S. y Tarpin-Bernard, F. (2010, octubre). Tools and methods for efficiently designing serious games. Proceedings of the 4th European Conference on Games Based Learning ECGBL (pp. 226-234).

Marne, B., Wisdom, J., Huynh-Kim-Bang, B. y Labat, J. (2012, septiembre). The six facets of serious game design: a methodology enhanced by our design pattern library. En A. Ravenscroft, S. Lindstaedt, C. D. Kloos y D. Hernández-Leo (Eds., 21st Century Learning for 21st Century Skills, 7.563, 208-221. https://doi.org/10.1007/978-3-642-332 63-0_17

Martin, A., Thompson, B. y Chatfield, T. (2006). Alternate reality games white paper. International Game Developers Association (IGDA). Christy Dena's Field Notes From Earth. 
http://www.christydena.com/wp-content/ uploads/2007/11/igda-alternaterealitygames-whitepaper-2006.pdf

McGonigal, J. (2003). This Is Not a Game: Immersive Aesthetics and Collective Play. https://janemcgonigal.files.wordpress. com/2010/12/mcgonigal-jane-this-is-not-agame.pdf

Morin, E. (2009). Introducción al pensamiento complejo. Gedisa.

OCDE. (2016). Revisión de políticas nacionales de educación. La educación en Colombia. (trad. Ministerio de Educación Nacional de Colombia). https://www.mineducacion.gov. co/1759/articles-356787_recurso_1.pdf

OCDE. (2019). Colombia-Country Note-PISA 2018 Results. https://www.oecd.org/pisa/ publications/PISA2018_CN_COL_ESP.pdf

Palmer, C. y Petroski, A. (2016). Alternate Reality Games: Gamification for Performance. CRC Press.

Ramírez, J. L. (2014). Gamificación: mecánicas de juegos en tu vida personal y profesional. SCLibro.

Rivas, A. (2015). América Latina después de PISA: lecciones aprendidas de la educación en siete países 2000-2015. Fundación CIPPEC. http://cippec.org/mapeal/wp-con tent/uploads/2015/05/Rivas_A_2015_Ame rica_Latina_despues_de_PISA.pdf

Ryan, B. (2014). Play hard, work harder. Game and scenario based learning in higher education. Proceedings from the International Conference on Engaging Pedagogy. Athlone Institute of Technology, Athlone, Irlanda. https://doi.org/10.21427/D7P9OH
Sanabria Janes, L. A., Pérez Almagro, M. ${ }^{a}$ C. y Riascos Hinestrosa, L. E. (2020). Pruebas de evaluación Saber y PISA en la educación obligatoria de Colombia. Educatio Siglo XXI, 38(3), 231-254. https://doi.org/10.6018/edu catio. 452891

UniCamacho. (2013). Modelo pedagógico institucional. https://issuu.com/comuniajc/docs/ modelo_pedag_gico_issu_protegido

Waddington, D. (2013). A parallel world for the World Bank: A case study of urgent: Evoke, an educational alternate reality game. Revue Internationale des Technologies en Pédagogie Universitaire/International Journal of Technologies in Higher Education, 10(3), 42-56. https://doi.org/10.7202/1035578ar

Watson, J. (2012). Reality Ends Here: Environmental Game Design and Participatory Spectacle (Tesis doctoral, University of Southern California).

Whitton, N. (2008, octubre). Alternatereality games for developing student autonomy and peer learning. Proceedings of the LICK 2008 Symposium (pp. 32-40). Manchester Metropolitan University's Research Repository. http:// e-space.mmu.ac.uk/id/eprint/144830

Young, J. (2010, enero 24). 5 teaching tips for professors-from video games. The Chronicle of Higher Education. https://www.chro nicle.com/article/5-Lessons-ProfessorsCan-Learn/63708

Zubiría Samper, J. de. (2006). Los modelos pedagógicos: hacia una pedagogía dialogante. Magisterio. 